\title{
Scaling and the center of band anomaly in a one-dimensional Anderson model with diagonal disorder
}

\author{
L. I. Deych ${ }^{a}$, M. V. Erementchouk ${ }^{a}$, A. A. Lisyansky ${ }^{a}$, and B. L. Altshuler ${ }^{b}$ \\ ${ }^{a}$ Physics Department, Queens College of the City University of New York, Flushing, NY 11367 \\ ${ }^{b}$ Physics Department, Princeton University and NEC Laboratories America, Princeton, NJ 08540
}

\begin{abstract}
We resolve the problem of the violation of single parameter scaling at the zero energy of the Anderson tight-binding model with diagonal disorder. It follows from the symmetry properties of the tight-binding Hamiltonian that this spectral point is in fact a boundary between two adjacent bands. The states in the vicinity of this energy behave similarly to states at other band boundaries, which are known to violate single parameter scaling.
\end{abstract}

\section{INTRODUCTION}

Despite a long history of research in the field of localization, even such a standard problem as the onedimensional Anderson tight-binding model with nearest neighbor hopping and diagonal disorder can still hold surprises. This model is described by the equation of motion

$$
\psi_{n+1}+\psi_{n-1}+\left(\epsilon_{n}-E\right) \psi_{n}=0,
$$

where, $\psi_{n}$ represents a wave function at the $n$-th site, $\epsilon_{n}$ is a random site energy distributed uniformly between $-W$ and $W$, and $E$ is the energy. Recently, Schomerus and Titov [1] demonstrated that single parameter scaling (SPS), which is a cornerstone of the conceptual framework for dealing with transport properties of disordered systems [2], is violated in this classical model of localization in the vicinity of the energy $E=0$. While the violation of SPS in the vicinities of the energies $E= \pm 2$ (which are band boundaries of the system with $W=0$, i.e., all $\epsilon_{n}=0$ ) is well established 3], the absence of SPS at the band center $E=0$ looks surprising. It is yet another manifestation of the anomalous nature of this spectral point. Whereas earlier studies of the Anderson model revealed an anomalous behavior of the localization length at $E=0[4,5,6,6]$, the violation of SPS in this spectral region remained unnoticed. The mathematical roots of the anomalous behavior of the localization length were established in Ref. 6. At the same time the physical understanding of the nature of the anomalous properties of $E=0$ is still absent. Here we suggest an approach to this spectral region based on symmetries of the Hamiltonian Eq. (11), which clarifies the physical nature of this anomaly, and explains scaling properties of the Anderson model in this spectral region.

It is convenient to describe the statistics of conductance, $g$, of a disordered chain of length $L$ in terms of the Lyapunov exponent (LE), $\tilde{\gamma}$, defined as [8]

$$
\tilde{\gamma}=\frac{1}{L} \ln \left(1+\frac{1}{g}\right) \text {. }
$$

SPS means that the probability distribution of $\tilde{\gamma}$ depends on a single parameter, so that, e.g., any moment of this distribution can be expressed through the mean value of LE, $\gamma \equiv\langle\tilde{\gamma}\rangle$. Such a relationship between $\gamma$ and the variance, $\sigma^{2} \equiv\left\langle\tilde{\gamma}^{2}\right\rangle-\langle\tilde{\gamma}\rangle^{2}$, was first conjectured in Ref. 8

$$
\tau \equiv \sigma^{2} L / \gamma=1 .
$$

The authors of Ref. 1 demonstrated that at $E=0$, parameter $\tau$ of Eq. (3) deviates from the SPS value and is equal to 1.047. This deviation from Eq. (3), which was overlooked by previous studies probably due to its small size, is of principle importance. It seemingly contradicts a well established criterion for SPS introduced in Ref. 3 .

In this Letter we show that this is only an apparent contradiction, and explain the violation of SPS at $E=0$ within the general picture developed in Ref. 3 . The anomaly at $E=0$ turns out to result from a hidden symmetry of the tight-binding model, which splits the conduction band into two adjacent bands with a boundary at $E=0$. This splitting does not affect thermodynamic properties of the model but is crucial to its scaling behavior.

Before presenting our resolution of the mysterious behavior in the vicinity of $E=0$, let us recall the history of the criterion for SPS. Originally, Anderson, et al. 8 suggested that SPS holds when the localization length of the system, $l_{l o c}=\gamma^{-1}$, exceeds what they called the phase randomization length: $l_{l o c}>l_{p h}$. The stationary distribution of the phases of the reflection and transmission amplitudes in disordered one-dimensional systems was assumed to become uniform as soon as the system length $L$ exceeds $l_{p h}$. Many authors derived Eq. (3) from this phase randomization hypothesis for several different models. The phase randomization itself was rigorously proven only for few particular models: banded matrices 9], the Anderson tight-binding model with diagonal disorder [6], and a continuous model with a white-noise random potential 10 for certain spectral regions. At the same time, it was shown that for some values of energies, such as $E=0$ or $E= \pm 2$ for the model described by Eq. (11) the stationary distribution of the phases is not uniform [4, 5, 6, 7, 11, 12].

Although in the vicinities of these special energies SPS is indeed violated 1, 13], the criterion for SPS based 
on the phase randomization length does not seem to describe the situation adequately. Indeed, the absence of the phase randomization in all situations discussed above does not mean that the "relaxation" length of the phase distribution diverges and exceeds the localization length when one approaches the respective energies. What actually happens is that the uniform contribution to the stationary phase distribution vanishes in the vicinity of these special energies. As a result, the nonuniform part, which always exists but is usually small, becomes dominant $[\underline{5}, 6,6]$. Thus, the phase randomization length, as it was introduced in Ref. [8], is ill-defined.

Thus, appealing to the phase distribution in connection with SPS simply substitutes the problem of formulating a criterion for SPS by the problem of finding a criterion for the uniform distribution of phases. Previously proposed criteria for the latter are specific for particular models. For instance, in Ref. 1 for the Anderson model within the white noise approximation, the criteria for the phase randomization for the band edges and the band center are set as $|E-2|>3 W^{2 / 3}$ and $|E|>10 D$, respectively ( $D$ is a measure of the disorder strength). These definitions do not look satisfactory to us. We believe that the criterion should be universal and formulated in terms of some fundamental macroscopic quantities.

Such a universal criterion for SPS similar in form to the Anderson's, $l_{l o c}>l_{p h}$, was recently suggested in Ref. 3:

$$
\kappa \equiv l_{l o c} / l_{s}>1 \text {. }
$$

The new length scale, $l_{s}$, has nothing to do with $l_{p h}$ and can be expressed through the integral density of states normalized by the total number of states in the band, $N(E)$, and a distance between the neighboring sites, $a$ :

$$
l_{s}=a / \sin [\pi N(E)] .
$$

This criterion was extracted from the exact calculation of the variance of LE for the Anderson model with the Cauchy distribution of the site energies, $\epsilon_{n}$ (the Lloyd model). Eq. (3) was derived in Ref. 3 without any relation to the phase distribution 17]. For in-band states, $N(E) \sim 1 / 2$ and $l_{s}$ is microscopic and therefore is not significant. For the states close to the band edges and fluctuation states in the former band gaps, where $N(E) \ll 1$ or $1-N(E) \ll 1, l_{s}$ becomes macroscopic and can exceed $l_{l o c}$. In the latter case, $l_{s}$ has a clear physical meaning as an average distance between localization centers responsible for the states with energies between $E$ and the closest fluctuation boundary of the spectrum.

The expression of $l_{s}$ in terms of the density of states $N(E)$ allowed authors of Refs. 3 to conjecture a generalization of this definition to other models. It was suggested that $N(E)$ in Eq. (5) must be understood as the total number of states (per unit length) between $E$ and a closest genuine spectral boundary normalized by the total number of states. This definition of $l_{s}$ can be used also in the case of systems with multiple bands, provided that the disorder does not fill the band gaps of the original systems completely, and there exist genuine spectral boundaries inside each of the band gaps. Under this condition, $N(E)$ refers to the number of states associated with one particular band of the spectrum, and varies between 0 and 1 when $E$ spans the states of the band.

In light of this criterion, one can understand the violation of SPS in the band gaps of the initial spectrum observed numerically in Ref. 13. Applicability of the $l_{s}$-based criterion was examined for several other models such as the Anderson model with the box [3] and dichotomic 14] distributions of the site energies, the model of a scalar wave propagating through a onedimensional disordered periodic-on-average superlattice, and even for the model of a wave propagating in an absorbing medium [15]. In all these cases, the criterion perfectly described the transition between the SPS behavior of in-band states, and non-SPS behavior of the band edge and fluctuation states. Moreover, as was shown recently in Ref. 16, the length $l_{s}$ plays an even more important role than simply determining a boundary between SPS and non-SPS spectral regions: the second and the third moments of the distribution of the Lyapunov exponent in the non-SPS region can be parameterized by a single parameter $\kappa$ provided that $L$ exceeds $l_{s}$ [18].

\section{THE BAND-CENTER ANOMALY IN THE ANDERSON MODEL}

The success of the scaling approach based on the parameter $\kappa$, Eqs. (4) and (5), in predicting violations of SPS at the band edges of different models, and in describing the probability distribution of LE in non-SPS spectral regions [3, 15, 16] motivated us to look more carefully at the reasons for the apparent failure of this approach at the center of the band of the Anderson model.

We start our analysis with a tight-binding model without disorder, $\epsilon_{n}=0$. The solution of Eq. (11) at $\epsilon_{n}=0$ is plane waves, $\psi_{n} \propto \exp ( \pm i k n a)$, with $k$ satisfying the dispersion equation

$$
E=2 \cos k a ; \quad 0 \leq k a \leq \pi,
$$

According to the traditional widely accepted point of view, Eq. (6) describes a single energy band $-2 \leq E \leq 2$,

However, this model contains more than the simple picture reveals. Indeed, the equations of motion, Eq. (10), at $\epsilon_{n}=0$ acquire an additional symmetry: Operation

$$
D_{n}=\psi_{n} \rightarrow(-1)^{n} \psi_{n}
$$

transforms a state $\psi_{n}(E)$ into the state $\psi_{n}(-E)$ : $\psi_{n}(E)=(-1)^{n} \psi_{n}(-E)$. This operation forms a group, and the states of this model can be classified according to its irreducible representations. 
In order to realize these representations, we introduce two field invariants with respect to $D_{n}$ :

$$
u_{l}=\psi_{2 l} ; \quad v_{l}=\psi_{2 l+1} .
$$

Solving equations of motion for these fields we find that the spectrum is separated into two branches described by the following dispersion equations

$$
E= \pm \cos (k a / 2) .
$$

Two bands of the spectrum described by Eq. (9) have a common boundary at $E=0$, and, therefore, the spectrum of the system appears as consisting of a single band, as it is usually assumed. However, the classification of the states according to the irreducible representations of $D_{n}$ reveals that $E=0$ should be considered as a band boundary rather than as a band center. The new description of the band structure can be viewed as a transition to the reduced zone representation, where the wave number of the original dispersion equation Eq. (6) is restricted to the interval $0 \leq k a \leq \pi / 2$. This operation is equivalent to doubling of the elementary cell of the original periodic chain. This can be justified by noticing that two adjacent sites of the chain are not equivalent with respect to the operation $D_{n}$ [Eq. [7] ].

The considerations presented above would have been merely an exercise, if the vicinity of $E=0$ did not behave anomalously in the presence of disorder. Of course, any disorder mixes states from $E<0$ and $E>0$ bands. However, since violation of SPS occurs in the vicinity of a spectral boundary of an unperturbed system [3], one can expect that $E=0$ behaves similarly to $E= \pm 2$ boundaries. Actually a similarity between properties of $E=0$ and other band boundaries was noted already in Ref. 6, where it was shown that the calculation of the LE in the vicinities of all three points $E= \pm 2$, and $E=0$ required almost identical mathematical approach.

The deviation of the parameter $\tau(\kappa)$, Eq. (3), from unity observed in Ref. 1] can be interpreted as a manifestation of the band-edge nature of the spectral point $E=0$. This deviation is, however, different from the behavior of $\tau(\kappa)$ in the vicinity of $E= \pm 2$. Near $E= \pm 2$, $\tau(\kappa)$ demonstrates a small overshoot above unity at the band side of these boundaries, and decreases quickly at the band gap sides [3]. Since two conduction bands are adjacent at $E=0$, there is no band gap related decrease of $\tau(\kappa)$. However, the band-edge nature of $E=0$ still manifests itself in the form of the overshoot, discovered in Ref. 1. This overshoot appears at both sides of $E=0$.

The fundamental question is whether the deviation from SPS near $E=0$ occurs in accordance with the criterion Eq. (4)? The answer is "yes", however, the definition of the length $l_{s}$, Eq. (5), should be modified to reflect the fact that the states are now distributed between two bands. Accordingly, when calculating $l_{s}$ for each of the bands, we have to keep in mind that $N(E)$

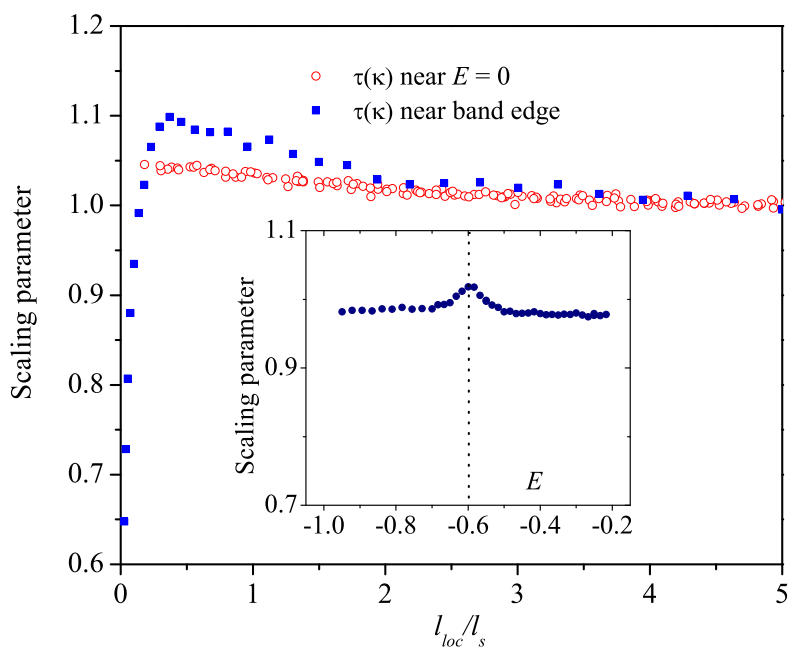

FIG. 1: Dependence of the scaling parameter $\tau$ on $\kappa$ in the vicinity of the fluctuating band edge $|E| \sim 2$ and near $E=0$ for different strengths of disorder: $W$ changes from 0.15 to 0.4. Curves corresponding to different strengths are indistinguishable. The inset shows $\tau(\kappa)$ for the Anderson model with next to the nearest neighbors interaction, Eq. (10), in the vicinity of the energy $E=-2 \alpha$ for $\alpha=0.3$.

in Eq. (5) is a number of states between $E=0$ and a given energy $E$ normalized by the total number of states in this band.

The results of the numerical calculations based on the new definition of $l_{s}$ reveal first of all (Fig. 11) that the criterion $\kappa>1$ and SPS are indeed violated simultaneously near $E=0$ as well as near $E=-2$. What we find even more important, however, is that the results of the calculations for different energies, strengths of disorder, and lengths of the system, all collapse to a single curve, when presented in the form $\tau(\kappa)$. Thus $\kappa \equiv l_{l o c} / l_{s}$, after the proper definition of $l_{s}$, remains a natural scaling variable for the entire spectrum of the system.

This means that separating negative and positive energies in two bands is physically significant. Of course, functions $\tau(\kappa)$ for the vicinities of $E=-2$ and $E=0$, do not coincide. This does not cause problems for our scaling description as long as these two regions are well separated by the spectral interval where $\tau=1$.

Additional arguments in favor of our interpretation of the $E=0$ anomaly can be obtained by modifying the initial Anderson model. For example, one can include next to the nearest neighbors interaction, and consider a model determined by the equation

$$
\psi_{n+1}+\psi_{n-1}+\alpha\left(\psi_{n+2}+\psi_{n-2}\right)+\left(\epsilon_{n}-E\right) \psi_{n}=0 .
$$

The spectrum of this model, in the absence of disorder, consists of two branches, only one of which corresponds to propagating states for $\alpha<1 / 4$. This branch is invariant with respect to a similar symmetry $(-1)^{n} \psi_{n}(E)=\psi_{n}\left(E^{\prime}\right)$. The stationary point of this transformation $E^{\prime}=E=-2 \alpha$ is a boundary between 


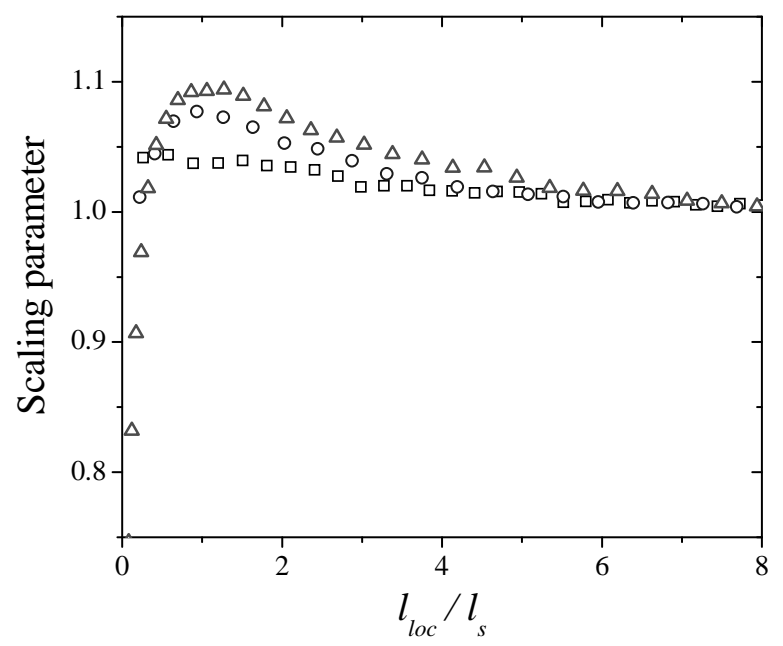

FIG. 2: Effect of the periodic potential on the scaling parameter $\tau$ near $E=0$ for $W=0.4$ Squares, circles and triangles correspond to $U_{0}=0.002,0.02$ and 0.2 , respectively.

two adjacent bands. Numerical calculations confirmed that indeed there is an anomaly in the dependence of the scaling parameter $\tau(\kappa)$ at $-2 \alpha$ similar to the one discussed above (see inset in Fig. 1).

To justify the physical significance of our treatment of $E=0$ as a band edge further we added a periodic potential $U_{n}=U_{0}(-1)^{n}$ to random site energies $\epsilon_{n}$. This potential breaks the symmetry of the model, and creates in the vicinity of $E=0$ a real band gap with a width proportional to $U_{0}$. The idea is to look at the sensitivity of the anomalous behavior in the vicinity of $E=0$ to the presence of the real band gap. This gap should decrease together with the magnitude of the symmetry-breaking potential. Will the features associated with the gap survive the vanishing of the gap width? The results of the numerical simulations answering this question are presented in Fig. 22 One can see that the anomalies in $\tau(\kappa)$ remain stable with respect to the symmetry-breaking perturbation. In the limit $U_{0} \rightarrow 0, \tau(\kappa)$ has the same behavior as for small but finite $U_{0}$. This observation in conjunction with the scaling arguments presented above gives strong support to our treatment of $E=0$ as a band edge.

Finally, there is one more question that should be addressed. Choosing an appropriate periodic potential one can create a band gap centered not only at $E_{\text {gap }}=0$ but anywhere in the spectrum. Why, then, is SPS violated only near $E=0$ ? It follows from our numerical studies that when the periodic potential is weak and $E_{\text {gap }} \neq 0$, the $\tau(\kappa)$-function always approaches the band edge without any overshoot above its SPS value, $\tau=1$; it simply decreases inside the gap. As a result, the normal SPS-like behavior is restored once the periodic potential vanishes. In the Lloyd model, the similar behavior is characteristic for all band boundaries, $E= \pm 2,0$. Therefore, the absence of a real band gap at $E=0$ masks the actual nature of this energy, and the center-band anomaly in the behavior of the second moment is not observed.

Concluding, we resolved the mystery of the anomalous properties in the vicinity of $E=0$ in the Anderson model with diagonal disorder. We demonstrated that due to an additional symmetry of the respective Hamiltonian, this spectral point must be considered as a band boundary between two adjacent bands rather than the center of a single band. The relevance of the criterion for SPS suggested in Refs. 3 is thus reestablished. Our approach allows one to describe statistical properties of conductance in the vicinity of $E=0$ within the framework of the scaling approach of Ref. 16.

The authors thank Steve Schwarz for reading and commenting on the manuscript. The work at Queens College was supported by AFOSR under Contract No. F4962002-1-0305, and partially by PSC-CUNY grants. BA gratefully acknowledges partial support by the EPSRC grant GR/R95432.

[1] H. Schomerus and M. Titov, Phys. Rev. B 67, 100201(R) (2003).

[2] E. Abrahams, P. W. Anderson, D. C. Licciardello, and T. V. Ramakrishnan, Phys. Rev. Lett. 42, 673 (1979).

[3] L. I. Deych, A. A. Lisyansky, and B. L. Altshuler, Phys. Rev. Lett. 84, 2678 (2000); Phys. Rev. B 64, 224202 (2001).

[4] M. Kappus and F Wegner, Z. Phys. B 45, 15 (1981).

[5] B. Derrida and E. Gardner, J. Physique 45, 1283 (1984).

[6] I. Goldhirsch, S. H. Noskowicz, and Z. Schuss, Phys. Rev. B 49, 14504 (1994).

[7] F. M. Izrailev, S. Ruffo, and L. Tessieri, J. Phys. A 31, 5263 (1998).

[8] P. W. Anderson, D. J. Thouless, E. Abrahams, and D. S. Fisher, Phys. Rev. B 22, 3519 (1980).

[9] Y. V. Fyodorov and A. D. Mirlin, JETP Lett. 58, 615 (1993); Int. J. Mod. Phys. B 8, 3795 (1994).

[10] H. Schomerus and M. Titov, Phys. Rev. E 66, 066207 (2002). preprint cond-mat/0204371 (2002).

[11] A. D. Stone, D. C. Allan, and J. D. Joanopoulos, Phys. Rev. B 27, 836 (1983).

[12] A. Ossipov, Tsampikos Kottos, and T. Geisel, Phys. Rev. B 61, 11411 (2000).

[13] L. I. Deych, D. Zaslavsky, and A. A. Lisyansky, Phys. Rev. Lett. 81, 5390 (1998).

[14] L. I. Deych, M.V. Erementchouk, and A. A. Lisyansky, Phys. Rev. B 67, 024205 (2003).

[15] L. I. Deych, A. Yamilov, and A. A. Lisyansky, Phys. Rev. B 64, 024201 (2001).

[16] L. I. Deych, M.V. Erementchouk, and A. A. Lisyansky, Phys. Rev. Lett. 90, 126601 (2003).

[17] The actual result for $\tau$ obtained in Ref. 3 differs by the factor of 2 from the standard form Eq. (3) because of the peculiarities of the Cauchy distribution

[18] For intermediate lengths, $l_{l o c} \ll L \ll l_{s}$, there is an additional parameter $L / l_{s}$, which describes an anomalous behavior of the second moment [16]. 